MODELING, IDENTIFICATION AND CONTROL, 1980, voL. 1, NO. 4, 231-245

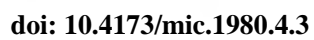

\title{
On the use of low-order Riccati equations in the design of a class of feedback controllers and state estimators
}

\author{
OLE A. SOLHEIM $\dagger$
}

Keywords: Riccati equation, optimal control, pole placement, state estimation.

\begin{abstract}
This paper describes a method for designing optimal feedback controllers with stability requirements using low-order Riccati equations. For originally stable systems the Riccati equation will be of first or second order, depending upon whether the eigenvalues are real or complex. For originally unstable systems the order of the Riccati equation will be equal to the number of eigenvalues with positive real part.

It is also shown how the method can be used to design optimal state estimators with prescribed stability.
\end{abstract}

\section{Introduction}

The paper presents a design method that may be considered as a combination of modal and optimal control, and it may be argued that it combines the 'best' features of both these methods.

The main drawback of the pure modal (pole-placement) method is that the design may be wasteful in control efforts or cost. That is, the controls may for example work against each other, while satisfying, of course, the eigenvalue specifications.

On the other hand, when using the optimal control method, one does not know the exact location of the eigenvalues beforehand. It may therefore happen that the controlled system, although minimizing the performance index, does not have the desired degree of stability.

As an alternative design method it could therefore be desirable to combine the two approaches, so that it may be possible to design a feedback controller that will give the system the specified eigenvalues and at the same time minimize a performance index.

To be more specific, the first problem we then are faced with, is to determine a $Q$-matrix (weighting matrix for the states) so that the closed-loop system attains the specified eigenvalues. Several methods exist for solving this problem. (See for example Tyler and Tuteur 1966, Chen and Shen 1969, Solheim 1972, Graupe 1972, Woodhead and Porter, Van de Vegte and Maki 1973.)

The purpose of the present paper is to show that if we use the method by Solheim (1972) to determine the $Q$-matrix, then we may solve the corresponding optimization problem by low-order Riccati equations.

Because of the duality between the optimal control problem and the optimal estimation problem, the presented method may also be used to design state estimators with prescribed stability and optimal use of the measurements.

$\dagger$ Division of Engineering Cybernetics, The Norwegian Institute of Technology, University of Trondheim, Trondheim, Norway. 


\section{Optimal feedback controllers}

\subsection{Problem statement}

Consider the linear dynamic system

$$
\dot{\boldsymbol{x}}=A \boldsymbol{x}+B u
$$

where $\boldsymbol{x}$ and $\boldsymbol{u}$ are, respectively, $\boldsymbol{n}$-dimensional state and $\boldsymbol{r}$-dimensional control vectors.

The usual quadratic performance index is to be used:

$$
J=\frac{1}{2} \int_{0}^{\infty}\left[x^{\mathrm{T}} Q x+u^{\mathrm{T}} P u\right] d t
$$

where $Q$ is a non-negative definite matrix and $P$ is a positive definite matrix.

The design method, to be presented here, is based on the fact that the controller in addition to minimizing the performance index, also shall give the closed-loop system

with the control law

$$
\dot{\boldsymbol{x}}=(A+B G) \boldsymbol{x}
$$

$$
\boldsymbol{u}=\boldsymbol{G} \boldsymbol{x}
$$

prescribed stability.

If the weighting matrices $Q$ and $P$ in the performance index are given, then the eigenvalues of the closed-loop system are uniquely determined. On the other hand, if we specify only $P$, the weighting matrix for the controls, then we may in addition specify the eigenvalues of the closed-loop system and find a $Q$-matrix that corresponds to these eigenvalues.

Using the maximum principle, we define the costate as

$$
\dot{\boldsymbol{p}}=-Q \boldsymbol{x}-\boldsymbol{A}^{\mathrm{T} p}
$$

The optimal control is given by the linear feedback control law

$$
u=-P^{-1} B^{\mathrm{T}} p=-P^{-1} B^{\mathrm{T}} R x=G x
$$

where $R$ is the solution of the stationary Riccati equation

$$
\dot{R}=-R A-A^{\mathrm{T}} R+R B P^{-1} B^{\mathrm{T}} R-Q=0
$$

Combining (1), (5) and (6) yields the canonical system

$$
\left[\begin{array}{c}
\dot{\boldsymbol{x}} \\
\dot{\boldsymbol{p}}
\end{array}\right]=\left[\begin{array}{c:c}
A & -B P^{-1} B^{\mathrm{T}} \\
\hdashline-Q & -A^{\mathrm{T}}
\end{array}\right]\left[\begin{array}{l}
x \\
p
\end{array}\right]=F\left[\begin{array}{l}
x \\
p
\end{array}\right]
$$

It is a well-known fact that this system has $n$ eigenvalues with negative real parts, and $n$ with positive real parts, and that the eigenvalues are located symmetrically about the imaginary axis. The eigenvalues of the optimal feedback system are identical to those eigenvalues of the canonical system that have negative real parts. It is therefore possible to study the eigenvalues of the canonical system instead of those of the feedback system. This has the great advantage that the eigenvalue dependence upon the weighting matrix, $Q$, may be studied without solving the Riccati equation.

It is necessary here to distinguish between several cases, depending upon whether the eigenvalues of the original process are real, distinct, complex or repeated. It is also necessary to distinguish between originally stable and unstable processes. 
In this paper, only processes with real distinct eigenvalues will be considered. For processes with complex or repeated eigenvalues we refer to Solheim (1979).

\subsection{Originally stable systems}

Using the transformation

$$
\boldsymbol{x}=M z
$$

where $M$ is an eigenvector matrix of the system matrix $A$, the system (1) is transformed into the diagonal form

$$
\dot{z}=\Lambda z+M^{-1} B u
$$

where $\Lambda$ is the diagonal eigenvalue matrix.

The costate of this diagonalized system becomes

where $\tilde{Q}=M^{\mathrm{T}} Q M$.

$$
\dot{\tilde{\boldsymbol{p}}}=-\tilde{Q} z-\Lambda \tilde{\boldsymbol{p}}
$$

In the following the tilde $(\sim)$ refers to the diagonalized system.

The optimal control in terms of the costate $\tilde{p}$ becomes

$$
\boldsymbol{u}=-P^{-1} B^{\mathrm{T}}\left(M^{-1}\right)^{\mathrm{T}} \tilde{\boldsymbol{p}}
$$

Combining (10), (11) and (12) yields the new canonical system

where

$$
\left[\begin{array}{c}
\dot{z} \\
\tilde{p}
\end{array}\right]=\left[\begin{array}{c:c}
\Lambda & -H \\
\hdashline-\tilde{Q} & -\Lambda
\end{array}\right]\left[\begin{array}{l}
z \\
\tilde{p}
\end{array}\right]=\tilde{F}\left[\begin{array}{l}
z \\
\tilde{p}
\end{array}\right]
$$

$$
H=M^{-1} B P^{-1} B^{\mathrm{T}}\left(M^{-1}\right)^{\mathrm{T}}
$$

The eigenvalues of $\widetilde{F}$ are identical to those of $F$ since these two systems can be shown to be similar.

Suppose now that the weighting matrix $\widetilde{Q}$ has only one non-zero element, namely $\tilde{q}_{j j}$. This means that only the mode $z_{j}$ is being considered in the performance index.

It can be shown (Solheim 1972) that the left half plane eigenvalues of $\widetilde{F}$ (and thus also of $F$ ) are

$$
\left.\begin{array}{l}
s_{i}=\lambda_{i}, \quad i \neq j, \quad i=1, \ldots, n \\
s_{j}=-\sqrt{ }\left(\lambda_{j}^{2}+\tilde{q}_{j j} h_{j j}\right)
\end{array}\right\}
$$

where $h_{j j}$ is an element of the $H$-matrix defined in (14).

Now, let $s_{j}$ be a prescribed eigenvalue of the optimal system. Then from (15)

$$
\tilde{q}_{j j}=\frac{s_{j}^{2}-\lambda_{j}^{2}}{h_{j j}}
$$

If the mode $z_{j}$ is controllable, it can be shown that $h_{j j}>0$.

With $\widetilde{Q}$ known, the optimal feedback gain can be obtained by solving the Riccati equation

$$
\dot{\tilde{R}}=-\widetilde{R} \Lambda-\Lambda \widetilde{R}+\widetilde{R} M^{-1} B P^{-1} B^{\mathrm{T}}\left(M^{-1}\right)^{\mathrm{T}} \widetilde{R}-\tilde{Q}=0
$$


There is, as will be shown below, only one non-zero element in the solution $\tilde{R}$, and consequently (17) reduces to a first order Riccati equation.

The optimal control can be obtained from (12) as

$$
u=-P^{-1} B^{\mathrm{T}}\left(M^{-1}\right)^{\mathrm{T}} \tilde{\mathrm{p}}=-P^{-1} B^{\mathrm{T}}\left(M^{-1}\right)^{\mathrm{T}} \widetilde{R} z=\tilde{G} z
$$

Now, since only mode $z_{j}$ is being considered and all modes are assumed stable, there can be feedback only from this mode, since feedback from any other mode would affect eigenvalues which are assumed not to be shifted.

Thus, we can write (18) as

$$
\boldsymbol{u}=\tilde{G} \boldsymbol{z}=\left[\begin{array}{c:c:c:c:c}
0 & 0 & \tilde{g}_{1 j} & 0 & 0 \\
- & - & - & - & - \\
- & - & - & - & - \\
- & - & - & - & - \\
- & \tilde{g}_{r j} & - & -
\end{array}\right] z
$$

From (18) we note that $\tilde{G}$ can be written as

$$
\tilde{G}=L \tilde{R}
$$

where $L$ may be a full matrix. Therefore, in order that $\tilde{G}$ obtains the form indicated in (19), we must require that $\widetilde{R}$, since it is symmetric, has the form

$$
\widetilde{R}=\left[\begin{array}{c:c:c:c}
0 & 0 & 0 & 0 \\
-- & - & - & - \\
\hline 0 & 0 & 0 & 0
\end{array}\right]
$$

Thus, the Riccati equation (17) can be simplified to

$$
h_{j j} \tilde{r}_{j j}^{2}-2 \lambda_{j} \tilde{r}_{j j}-\tilde{q}_{j j}=0
$$

where $\tilde{q}_{j j}$ is given by (16) and $h_{j j}$ is the same element as in (16).

The solution of (22) is

$$
\tilde{r}_{j j}=\left[\lambda_{j}+\sqrt{ }\left(\lambda_{j}{ }^{2}+\tilde{q}_{j j} h_{j j}\right)\right] / h_{j j}
$$

where, since $\lambda_{j} \leqslant 0$, we have chosen the plus sign before the root in order that $\tilde{r}_{j j}$ becomes non-negative.

Substituting for $\tilde{q}_{j j}$ yields the very simple solution to the Riccati equation

$$
\underline{\tilde{r}_{j j}=\frac{\lambda_{j}-s_{j}}{h_{j j}}}
$$

Having thus obtained $\tilde{R}$, the feedback is computed from (18):

$$
\underline{G=\tilde{G} M^{-1}=-P^{-1} B^{\mathrm{T}}\left(M^{-1}\right)^{\mathrm{T}} \tilde{R} M^{-1}}
$$

In the optimal feedback system

$$
\dot{\boldsymbol{x}}=(A+B G) \boldsymbol{x}
$$

we have now shifted one eigenvalue of the open-loop system to its specified position. We may now start with the new system $A_{1}=(A+B G)$ and shift the next eigenvalue. 
The result is a recursive procedure. The sequence in which the eigenvalues are shifted may be arbitrary but a different sequence will usually give a different final feedback. We should mention that the feedback controller, $G$, that will minimize the performance index with a given $P$-matrix and give the system prescribed eigenvalues, is, in general, not unique.

\subsection{Originally unstable systems}

In this case we shall first determine a feedback controller $G_{0}$ so that the positive eigenvalues are shifted symmetrically about the imaginary axis into the left half plane. In order to do this, we use a weighting matrix $Q_{0}=0$.

Let us assume for the time being that there is only one unstable mode $z_{l}$. In order to shift the corresponding positive eigenvalue $\lambda_{l}$ into the left half plane we need a feedback

or

$$
u=\tilde{G}_{0} z=\left[\begin{array}{c:c:c:c:c}
0 & 0 & \tilde{g}_{11} & 0 & 0 \\
-- & - & - & - & - \\
\hdashline- & - & - & - & - \\
0 & 0 & \tilde{g}_{r l} & 0 & 0
\end{array}\right] z
$$

$$
u_{j}=g_{j i} z_{i} \quad j=1, \ldots, r
$$

We note that the feedback controller $\tilde{G}_{0}$ should affect the unstable mode $z_{l}$ only.

Using the maximum principle we get

$$
\tilde{G}_{0}=-P^{-1} B^{\mathrm{T}}\left(M^{-1}\right)^{\mathrm{T}} \tilde{R}_{0}=K \tilde{R}_{0}
$$

where $\widetilde{R}_{0}$ is the solution of the Riccati equation

$$
\hat{R}_{0}=-\tilde{R}_{0} \Lambda-\Lambda \widetilde{R}_{0}+\tilde{R}_{0} M^{-1} B P^{-1} B^{\mathrm{T}}\left(M^{-1}\right)^{\mathrm{T}} \tilde{R}_{0}=0
$$

Note that in this case $\tilde{Q}=M^{\mathrm{T}} Q M=0$.

Using the same arguments as above for stable systems, there can be only one non-zero element, $\tilde{r}_{u}$, in $\widetilde{R}_{0}$. Thus, eqn. (30) can be simplified to the first order Riccati equation.

$$
h_{i i}\left(\tilde{r}_{i i}\right)^{2}-2 \lambda_{i} \tilde{r}_{i i}=0
$$

where $h_{i i}$ is an element of the matrix

$$
H=M^{-1} B P^{-1} B^{\mathrm{T}}\left(M^{-1}\right)^{\mathrm{T}}
$$

As mentioned above, it can be shown that if the mode $z_{i}$ is controllable, then $h_{l l}>0$.

The solution of (31) is

$$
\tilde{r}_{i t}=0 \quad \text { or } \quad \tilde{r}_{i t}=\frac{2 \lambda_{i}}{h_{i l}}
$$

Since $\lambda_{l}>0$ we choose the positive solution

$$
\tilde{r}_{i l}=\frac{2 \lambda_{l}}{h_{u l}}
$$

(Note that if $\lambda_{l}<0$ (which, however, contradicts our assumption here) we would have to choose the non-negative solution $\tilde{r}_{i t}=0$, which would result in $\tilde{G}_{0}=0$ ). 
Having solved the Riccati equation, $\widetilde{G}_{0}$ can be computed from (29). The control $\boldsymbol{u}$ becomes

$$
u=\widetilde{G}_{0} z=\widetilde{G}_{0} M^{-1} x=G_{0} x
$$

The feedback control is thus

$$
G_{0}=\widetilde{G}_{0} M^{-1}
$$

The new system becomes

$$
\dot{\boldsymbol{x}}=\left(A+B G_{0}\right) x=A_{0} x
$$

where $A_{\mathrm{o}}$ has no eigenvalues in the right half plane.

If there is more than one unstable mode, the order of the Riccati equation for determining the feedback to stabilize the system will be equal to the number of unstable modes. Let us number the $k$ unstable eigenvalues

In this case we get

$$
\lambda_{1}, \lambda_{2}, \ldots, \lambda_{k}
$$

$$
\tilde{R}_{0}=\left[\begin{array}{c:c}
\left(\widetilde{R}_{k}\right)_{0} & 0 \\
\hdashline 0 & 0
\end{array}\right]
$$

where $\left(\tilde{R}_{k}\right)_{0}$ is determined from the $k$ th-order Riccati equation

$$
\left(\widetilde{R}_{k}\right)_{0} \Lambda_{k}+\Lambda_{k}\left(\widetilde{R}_{k}\right)_{0}-\left(\widetilde{R}_{k}\right)_{0} H_{k}\left(\widetilde{R}_{k}\right)_{0}=0
$$

where

$$
\Lambda_{k}=\left[\begin{array}{ccc}
\lambda_{1} & & 0 \\
& \ddots & \\
0 & & \lambda_{k}
\end{array}\right]
$$

and $H_{k}$ is the following $k \times k$ submatrix of the $H$-matrix defined in (32):

$$
H=\left[\begin{array}{c:c}
H_{k} & x \\
\hdashline x & x
\end{array}\right]
$$

\subsection{Computational procedure}

Given: $A, B, P$, prescribed eigenvalues of closed-loop system.

1. Compute

$$
\Lambda, M_{0}, H_{0}=M_{0}^{-1} B P^{-1} B^{\mathrm{T}}\left(M_{0}^{-1}\right)^{\mathrm{T}}
$$

2. If all

$$
\lambda_{j} \leqslant 0, \quad i=1, \ldots, n \text { set } i=1, M_{1}=M_{0}, H_{1}=H_{0}, A_{0}=A
$$

and go to 4 .

3. Assume $\lambda_{j}>0, j=1, \ldots, k$.

Solve the $k$ th-order Riccati equation (38) (or (33) if $k=1$ ) and compute the feedback $G_{0}=-P^{-1} B^{\mathrm{T}}\left(M_{0}^{-1}\right)^{\mathrm{T}} \widetilde{R}_{0} M_{0}^{-1}$ where $\widetilde{R}_{0}$ is given in (37).

Set $A_{0}=A+B G_{0}, G=G_{0}$ and go to 7 . 
4. The eigenvalue $\lambda_{j}$ is to be shifted to $s_{j}\left(s_{j}<\lambda_{j}\right)$.

Compute

Compute

$$
\begin{aligned}
& \left(\tilde{q}_{j j}\right)_{i}=\frac{s_{j}{ }^{2}-\lambda_{j}{ }^{2}}{\left(h_{j j}\right)_{i}} \\
& \tilde{Q}_{i}=\left[\begin{array}{c:c:c:c:c}
0 & 0 & 0 & 0 & 0 \\
- & - & - & - & - \\
\left.-\overline{q_{j j}}\right)_{j} & - & - & - \\
0 & 0 & 0 & 0 & 0
\end{array}\right]
\end{aligned}
$$

$$
\begin{aligned}
& \left(\tilde{r}_{j j}\right)_{i}=\frac{\lambda_{j}-s_{j}}{\left(h_{j j}\right)_{i}} \\
& \tilde{R}_{i}=\left[\begin{array}{c:c:c:c:c}
0 & 0 & 0 & 0 & 0 \\
-- & - & - & - & - \\
\hdashline 0 & \left.-\tilde{r}_{j j}\right)_{i} & - & - \\
0 & 0 & 0 & 0
\end{array}\right]
\end{aligned}
$$

5. Compute:

$$
\begin{aligned}
G_{i} & =-P^{-1} B^{\mathrm{T}}\left(M_{i}^{-1}\right)^{\mathrm{T}} \widetilde{R}_{i} M_{i}^{-1} \\
Q_{i} & =\left(M_{i}^{-1}\right)^{\mathrm{T}} \widetilde{Q}_{i} M_{i}^{-1} \\
G & =G+G_{i} \\
Q & =Q+Q_{i} \\
A_{i} & =A+B G
\end{aligned}
$$

6. If more eigenvalues are to be shifted, then change $j$ and go to 7 , otherwise the program is finished.

7. $i=i+1$

Compute $M_{i}$ (based on $A_{i-1}$ ) and $H_{i}$. Go to 4 .

\section{Optimal state estimators}

\subsection{Problem statement}

Consider the system

$$
\left.\begin{array}{l}
\dot{x}=A x+C v \\
y=D x+w
\end{array}\right\}
$$

where $\boldsymbol{x}$ is the $n$-dimensional state vector, $\boldsymbol{y}$ the $m$-dimensional output (or measurement) vector, $\boldsymbol{v}$ the $s$-dimensional process noise vector and $\boldsymbol{w}$ the $m$-dimensional measurement noise vector. Assuming white noise with zero mean, we denote the covariance matrices for the process and measurement noise by $V$ and $W$ respectively.

The state estimator to be designed is described by

$$
\dot{\hat{x}}=A \hat{\boldsymbol{x}}+K(\boldsymbol{y}-D \hat{x})=(A-K D) \hat{\boldsymbol{x}}+K \boldsymbol{y}
$$

where the symbol ${ }^{\wedge}$ indicates estimated quantities. $K$ is the estimator gain matrix. 
This estimation problem is the dual of the optimal control problem presented in $\S 2$. For example, if we in the control system are aiming at an optimal use of the control efforts, the dual is that we in the estimator try to use the measurements in an optimal way. This means again that we are weighing measurements that contain little noise more than those that contain much noise.

Now, the dual to the cost matrix $P$ for the controls is the covariance matrix $W$ for the measurement noise. The dual to the weighting matrix $Q$ is the covariance matrix $V$ for the process noise.

Based on this duality between optimal control and optimal estimation, it is now possible to use the technique described in $\S 2$ to design an optimal estimator. The dual of finding a $Q$-matrix that will give the specified stability in the controller case is that of finding a $V$-matrix that will give the specified stability in the estimator case.

For an optimal estimator the gain matrix is given by (see for example Bryson and Ho 1969)

$$
K=R D^{\mathrm{T}} W^{-1}
$$

where $R$ is the solution of the stationary Riccati equation

$$
\dot{R}=A R+R A^{\mathrm{T}}-R D^{\mathrm{T}} W^{-1} D R+C V C^{\mathrm{T}}=0
$$

The canonical system matrix (the dual to (8)) becomes

$$
F_{\mathrm{E}}=\left[\begin{array}{c:c}
-A^{\mathrm{T}} & -D^{\mathrm{T}} W^{-1} D \\
\hdashline-C V C^{\mathrm{T}} & A
\end{array}\right]
$$

The eigenvalues of the optimal estimator $(A-K D)$ are identical to the eigenvalues with negative real part of the canonical system $F_{\mathrm{E}}$. The basis for the design of the estimator is that the eigenvalues of $(A-K D)$ are specified.

As for the optimal controller case, we will also consider here systems with real, distinct eigenvalues only.

\subsection{Originally stable systems}

Diagonalization of the system (39) through the transformation $x=M z$ yields

$$
\left.\begin{array}{l}
\dot{z}=\Lambda z+M^{-1} C v \\
y=D M z+w
\end{array}\right\}
$$

The new canonical system becomes:

$$
\tilde{F}_{\mathrm{E}}=\left[\begin{array}{c:c}
-\Lambda & -H_{\mathrm{E}} \\
\hdashline-\tilde{V} & \Lambda
\end{array}\right]
$$

where

$$
\begin{aligned}
H_{\mathrm{E}} & =M^{\mathrm{T}} D^{\mathrm{T}} W^{-1} D M \\
\tilde{V} & =M^{-1} C V C^{\mathrm{T}} M^{-\mathrm{T}}
\end{aligned}
$$


The eigenvalues of the canonical system $\widetilde{F}_{\mathrm{E}}$ are obtained from:

$$
\left|\begin{array}{c:c}
s+\Lambda & H_{\mathbf{E}} \\
\hdashline V & s-\Lambda
\end{array}\right|=0
$$

We now proceed in exactly the same way as was mentioned in $\S 2$, except that we now have to replace the performance matrix $Q$ by the covariance matrix $V$. Assuming that

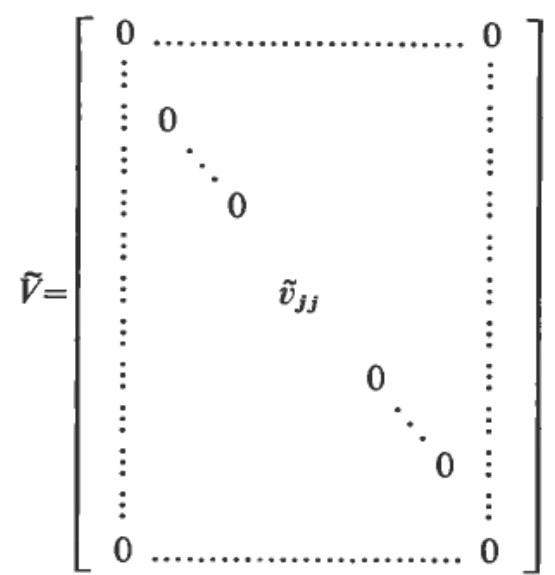

then

$$
\underline{\tilde{v}_{j j}=\frac{s_{j}^{2}-\lambda_{j}^{2}}{\left(h_{j j}\right)_{\mathrm{E}}}}
$$

where the eigenvalue $\lambda_{j}$ is being shifted to $s_{j}$, and $\left(h_{j j}\right)_{\mathrm{E}}$ is an element of the $H_{\mathrm{E}^{-}}$ matrix defined in (45).

If the mode $z_{j}$ is not observable, then the $j$ th column of $D M$ has zero elements only, so that in this case $\left(h_{j j}\right)_{\mathrm{E}}=0$.

With $\tilde{v}_{j j}$ known we solve the Riccati equation

$$
\tilde{R}=\Lambda \tilde{R}+\tilde{R} \Lambda-\tilde{R} M^{\mathrm{T}} D^{\mathrm{T}} W^{-1} D M \tilde{R}+\tilde{V}=0
$$

The optimal gain for the estimator of the diagonalized system is

$$
\widetilde{K}=\widetilde{R} M^{\mathrm{T}} D^{\mathrm{T}} W^{-1}
$$

This estimator is described by the equation:

$$
\hat{z}=\Lambda \hat{z}-\tilde{K} D M \hat{z}+\tilde{K} y
$$

Replacing $\hat{z}$ with $\hat{\boldsymbol{x}}$, we obtain:

$$
\begin{aligned}
M^{-1} \hat{\mathbf{x}} & =\Lambda M^{-1} \hat{\boldsymbol{x}}-\tilde{K} D \hat{\boldsymbol{x}}+\tilde{K} \boldsymbol{y}, \\
\hat{\boldsymbol{x}} & =A \hat{\boldsymbol{x}}-M \tilde{K} D \hat{\boldsymbol{x}}+M \tilde{K} \boldsymbol{y}=[A-K D] \hat{\boldsymbol{x}}+K \boldsymbol{y}
\end{aligned}
$$

where $K=M \tilde{K}$.

The eigenvalues of the estimator $[A-K D]$ will be the same as those of $[\Lambda-\widetilde{K} D M]$ since these matrices are similar. 
Since we are shifting the eigenvalue $\lambda_{j}$ only, then the mode $z_{j}$, corresponding to this eigenvalue, is the only mode to be affected by the feedback through $K$. Using the same arguments as in $\S 2$, we conclude that the solution of the Riccati equation (49) can have one non-zero element only, namely $\tilde{r}_{j J}$. Equation (49) thus simplifies to the first-order equation

$$
\left(h_{j j}\right)_{\mathrm{E}} \tilde{r}_{j j}^{2}-2 \lambda_{j} \tilde{r}_{j j}-\tilde{v}_{j j}=0
$$

Solving this equation and substituting for $\tilde{v}_{j j}$ from (48) yields the very simple solution to the Riccati equation

$$
\underline{\tilde{r}_{j j}=\frac{\lambda_{j}-s_{j}}{\left(h_{j j}\right)_{\mathrm{E}}}}
$$

Having thus obtained $\widetilde{R}$, the estimator gain matrix $K$ is computed from (50) and (53).

In the estimator $(A-K D)$ we have now shifted one eigenvalue to its specified position. We may now continue with the other eigenvalues in the same way as we did for the feedback control in $\S 2$.

\subsection{Originally unstable systems}

In order to use the technique described above for unstable systems, we first have to shift the right half plane eigenvalues into the left half plane, as we also did in the optimal controller case. This is done by setting $V=0$ and by solving a Riccati equation of order equal to the number of unstable modes.

\subsection{Computational procedure}

Given $A, C, D, W$, prescribed eigenvalues of estimator.

1. Compute

$$
\Lambda, M_{0},\left(H_{\mathrm{E}}\right)_{0}=M_{0}^{\mathrm{T}} D^{\mathrm{T}} W^{-1} D M_{0}
$$

2. If all

$$
\lambda_{j} \leqslant 0, \quad j=1,2, \ldots, n \text { set } i=1, M_{1}=M_{0},\left(H_{1}\right)_{\mathrm{E}}=\left(H_{0}\right)_{\mathrm{E}}
$$

$\left(A_{\mathrm{O}}\right)_{\mathrm{E}}=A$ and go to 4 .

3. Assume $\lambda_{j}>0, j=1, \ldots, k$.

Solve a $k$ th-order Riccati equation based on (49) with $\tilde{V}=0$ and developed in the same way as (38). Compute the estimator gain

$$
K_{0}=M_{0} \tilde{R}_{0} M_{0}^{\mathrm{T}} D^{\mathrm{T}} W^{-1} .
$$

Set $\left(A_{\mathrm{0}}\right)_{\mathrm{E}}=A-K_{\mathrm{0}} D, K=K_{\mathrm{0}}$ and go to 7 .

4. The eigenvalue $\lambda_{j}$ is to be shifted to $s_{j}\left(s_{j}<\lambda_{j}\right)$

Compute

Compute

$$
\left(\tilde{v}_{j j}\right)_{i}=\frac{s_{j}^{2}-\lambda_{j}^{2}}{\left(h_{j j}\right)_{\mathrm{E}}, i}
$$

$$
\left(\tilde{r}_{j j}\right)_{i}=\frac{\lambda_{j}-s_{j}}{\left(h_{j j}\right)_{\mathrm{E}}, i}
$$


5. Compute

$$
\begin{aligned}
K_{i} & =M_{i} \tilde{R}_{i} M_{i}^{\mathrm{T}} D^{\mathrm{T}} W^{-1} \\
C V_{i} C^{\mathrm{T}} & =M_{i} \widetilde{V}_{i} M_{i}^{\mathrm{T}} \\
K & =K+K_{i} \\
C V C^{\mathrm{T}} & =C V C^{\mathrm{T}}+C V_{i} C^{\mathrm{T}} \\
\left(A_{i}\right)_{\mathrm{E}} & =A-K D
\end{aligned}
$$

6. If more eigenvalues are to be shifted, then change $j$ and go to 7 , otherwise the program is finished.

7. $i=i+1$

Compute $M_{i}$ (based on $\left.\left(A_{i-1}\right)_{\mathrm{E}}\right)$ and $\left(H_{i}\right)_{\mathrm{E}}$. Go to 4 .

\section{Numerical examples}

\subsection{Optimal feedback controller}

Given the system

$$
\begin{gathered}
A=\left[\begin{array}{cc}
-2 & 0 \\
1 & 1
\end{array}\right], \quad B=\left[\begin{array}{ll}
1 & 0 \\
0 & 1
\end{array}\right], \quad P=\left[\begin{array}{ll}
1 & 0 \\
0 & 5
\end{array}\right] \\
\lambda_{1}=-2, \quad \lambda_{2}=+1
\end{gathered}
$$

Eigenvalues of the optimal closed loop system are specified as

$$
s_{1}=-8, \quad s_{2}=-5
$$

We compute

$$
M_{0}=\left[\begin{array}{cc}
3 & 0 \\
-1 & 1
\end{array}\right], \quad M_{0}^{-1}=\left[\begin{array}{cc}
1 / 3 & 0 \\
1 / 3 & 1
\end{array}\right], \quad H_{0}=\left[\begin{array}{cc}
1 / 9 & 1 / 9 \\
1 / 9 & 14 / 45
\end{array}\right]
$$

In order to make the system stable we first shift the unstable eigenvalue +1 to -1 . This is done by the feedback $G_{0}=-P^{-1} B^{\mathrm{T}}\left(M_{0}^{-1}\right)^{\mathrm{T}} \widetilde{R}_{0} M_{0}^{-1}$ where $\widetilde{R}_{0}$ is the solution of the Riccati equation (33). We get

$$
\tilde{r}_{22}=\frac{2 \lambda_{2}}{\left(h_{22}\right)_{0}}=\frac{2}{14 / 15}=6.43
$$

or

$$
\begin{aligned}
& \tilde{R}_{\mathrm{o}}=\left[\begin{array}{ll}
0 & 0 \\
0 & 6 \cdot 43
\end{array}\right] \\
& G_{0}=\left[\begin{array}{ll}
-5 / 7 & -15 / 7 \\
-3 / 7 & -9 / 7
\end{array}\right]
\end{aligned}
$$


The eigenvalues of the system

are now -2 and -1 .

$$
A_{\mathrm{o}}=\left[A+B G_{\mathrm{o}}\right]=\left[\begin{array}{cc}
-19 / 7 & -15 / 7 \\
4 / 7 & -2 / 7
\end{array}\right]
$$

We compute for this new system

$$
\begin{aligned}
M_{1} & =\left[\begin{array}{cc}
3 & 1 \\
-1 & -4 / 5
\end{array}\right] \\
M_{1}^{-1} & =\left[\begin{array}{cc}
4 / 7 & 5 / 7 \\
-5 / 7 & -15 / 7
\end{array}\right], H=\frac{1}{49}\left[\begin{array}{cc}
21 & -35 \\
-35 & 70
\end{array}\right]
\end{aligned}
$$

We now shift the eigenvalue at -2 to -8 . Using (24):

$$
\begin{aligned}
&\left(\tilde{r}_{11}\right)_{1}=\frac{\lambda_{1}-s_{j}}{\left(h_{11}\right)_{1}}=\frac{-2-(-8)}{21 / 49}=14 \\
& \tilde{R}_{1}=\left[\begin{array}{cc}
14 & 0 \\
0 & 0
\end{array}\right] \\
& G_{1}=\left[\begin{array}{cc}
-32 / 7 & -40 / 7 \\
-8 / 7 & -10 / 7
\end{array}\right] \\
& A_{1}=A_{0}+B G_{1}=\left[\begin{array}{cc}
-51 / 7 & -55 / 7 \\
-4 / 7 & -12 / 7
\end{array}\right]
\end{aligned}
$$

The eigenvalues of $A_{1}$ are now -8 and -1 .

The corresponding $\widetilde{Q}_{1}$ is obtained from (16):

$$
\begin{gathered}
\left(\tilde{q}_{11}\right)_{1}=\frac{s_{1}{ }^{2}-\lambda_{1}{ }^{2}}{\left(h_{11}\right)_{1}}=\frac{(-8)^{2}-(-2)^{2}}{21 / 49}=60 \\
\tilde{Q}_{1}=\left[\begin{array}{cc}
60 & 0 \\
0 & 0
\end{array}\right] \\
Q_{1}=\left(M_{1}^{-1}\right)^{\mathrm{T}} \tilde{Q}_{1} M_{1}^{-1}=\frac{1}{49}\left[\begin{array}{cc}
960 & 1200 \\
1200 & 1500
\end{array}\right]
\end{gathered}
$$

Next we shift the eigenvalue at -1 to -5 .

$$
M_{2}=\left[\begin{array}{cc}
11 & 1 \\
1 & -4 / 5
\end{array}\right], M_{2}{ }^{-1}=\frac{1}{49}\left[\begin{array}{cc}
4 & 5 \\
4 & -55
\end{array}\right], H_{2}=\frac{1}{343}\left[\begin{array}{cc}
3 & -5 \\
-5 & 90
\end{array}\right]
$$


Using again (24):

$$
\begin{gathered}
\left(\tilde{r}_{22}\right)_{2}=\frac{-1-(-5)}{90 / 343}=686 / 45 \\
\widetilde{R}_{2}=\left[\begin{array}{cc}
0 & 0 \\
0 & 286 / 45
\end{array}\right] \\
G_{2}=\frac{1}{63}\left[\begin{array}{cc}
-10 & 110 \\
22 & -242
\end{array}\right] \\
A_{2}=A_{1}+B G_{2}=\frac{1}{63}\left[\begin{array}{cc}
-469 & -385 \\
-14 & -350
\end{array}\right]
\end{gathered}
$$

The eigenvalues of $A_{2}$ are now the specified -8 and -5 .

$\widetilde{Q}_{2}$ is obtained from (16):

$$
\begin{aligned}
\left(\tilde{q}_{22}\right)_{2} & =\frac{(-5)^{2}-(-1)^{2}}{90 / 343}=1372 / 15 \\
\tilde{Q}_{2} & =\left[\begin{array}{cc}
0 & 0 \\
0 & 1372 / 15
\end{array}\right] \\
Q_{2} & =\left(M_{2}^{-1}\right)^{\mathrm{T}} \tilde{Q}_{2} M_{2}^{-1}
\end{aligned}
$$

The final $Q$-matrix becomes

$$
Q=Q_{1}+Q_{2}=\left[\begin{array}{cc}
20 \cdot 54 & 14 \cdot 01 \\
14 \cdot 01 & 145 \cdot 85
\end{array}\right]
$$

The final feedback controller becomes

$$
G=G_{0}+G_{1}+G_{2}=\left[\begin{array}{cc}
-5.44 & -6.11 \\
-1.22 & -6.56
\end{array}\right]
$$

\subsection{Optimal state estimator}

Given the system

$$
A=\left[\begin{array}{cc}
-2 & 0 \\
1 & -1
\end{array}\right], \underset{W=[1]}{D=\left[\begin{array}{ll}
0 & 1
\end{array}\right], \quad C=\left[\begin{array}{ll}
1 & 0 \\
0 & 1
\end{array}\right],}
$$

We compute

$$
M_{1}=\left[\begin{array}{cc}
1 & 0 \\
-1 & 1
\end{array}\right], \quad\left(H_{\mathrm{E}}\right)_{1}=M_{1}^{\mathrm{T}} D^{\mathrm{T}} W^{-1} D M_{1}=\left[\begin{array}{cc}
1 & -1 \\
-1 & 1
\end{array}\right]
$$


The eigenvalues of the process are $\lambda_{1}=-2$ and $\lambda_{2}=-1$. Let the specified eigenvalues of the estimator be $s_{1}=-8$ and $s_{2}=-5$.

Let us start by shifting the eigenvalue at -1 to -5 . From (48):

$$
\left(\tilde{v}_{22}\right)_{1}=24
$$

or

$$
\begin{gathered}
\tilde{V}_{1}=\left[\begin{array}{ll}
0 & 0 \\
0 & 24
\end{array}\right] \\
C V_{1} C^{\mathrm{T}}=M_{1} \tilde{V}_{1} M_{1}^{\mathrm{T}}=\left[\begin{array}{ll}
0 & 0 \\
0 & 24
\end{array}\right]
\end{gathered}
$$

From (55):

$$
\left(\tilde{r}_{22}\right)_{1}=\frac{\lambda_{2}-s_{2}}{\left(h_{22}\right)_{\mathrm{E}, 1}}=\frac{-1+5}{1}=4
$$

Thus

$$
\tilde{R}_{1}=\left[\begin{array}{ll}
0 & 0 \\
0 & 4
\end{array}\right]
$$

Using (50) and (53)

$$
K_{1}=M_{1} \widetilde{K}_{1}=M_{1} \widetilde{R}_{1} M_{1}^{\mathrm{T}} D^{\mathrm{T}} W^{-1}=\left[\begin{array}{l}
0 \\
4
\end{array}\right]
$$

We get the system

$$
\left(A_{1}\right)_{\mathrm{E}}=A-K_{1} D=\left[\begin{array}{cc}
-2 & 0 \\
1 & -5
\end{array}\right]
$$

We compute based on $\left(A_{1}\right)_{\mathrm{E}}$ :

$$
M_{2}=\left[\begin{array}{ll}
3 & 0 \\
1 & 1
\end{array}\right], \quad\left(H_{\mathrm{E}}\right)_{2}=\left[\begin{array}{ll}
1 & 1 \\
1 & 1
\end{array}\right]
$$

Next we shift the eigenvalue at -2 to the specified value -8 .

Using again (48):

$$
\left(\tilde{v}_{11}\right)_{2}=60
$$

giving

$$
\begin{aligned}
\tilde{V}_{2} & =\left[\begin{array}{cc}
60 & 0 \\
0 & 0
\end{array}\right] \\
C V_{2} C^{\mathrm{T}} & =\left[\begin{array}{cc}
540 & 180 \\
180 & 60
\end{array}\right]
\end{aligned}
$$


The solution to the Riccati equation is again obtained from (55)

Thus

$$
\left(\tilde{r}_{11}\right)_{2}=\frac{\lambda_{1}-s_{1}}{\left(h_{11}\right)_{\mathrm{E}, 2}}=\frac{-2+8}{1}=6
$$

$$
\widetilde{R}_{2}=\left[\begin{array}{ll}
6 & 0 \\
0 & 0
\end{array}\right]
$$

Using (50) and (53)

$$
K_{2}=M_{2} \tilde{K}_{2}=M_{2} \tilde{R}_{2} M_{2}^{\mathrm{T}} D^{\mathrm{T}} W^{-1}=\left[\begin{array}{c}
18 \\
6
\end{array}\right]
$$

The final result is

$$
\begin{gathered}
C V C^{\mathrm{T}}=C\left(V_{1}+V_{2}\right) C^{\mathrm{T}}=\left[\begin{array}{rr}
540 & 180 \\
180 & 84
\end{array}\right] \\
K=K_{1}+K_{2}=\left[\begin{array}{c}
18 \\
10
\end{array}\right]
\end{gathered}
$$

The eigenvalues of the estimator $(A-K D)$ are now as specified.

\section{Concluding remarks}

A method has been presented which permits the design of optimal controllers with stability requirements using low-order Riccati equations. For originally stable systems the Riccati equation will be of first or second order, depending upon whether the eigenvalues are real or complex. For originally unstable systems the order of the Riccati equation will be equal to the number of eigenvalues with positive real part.

Since the state estimation problem can be considered to be the dual of the optimal control problem, the method can also be used to design optimal state estimators with prescribed stability.

\section{REFERENCES}

Bryson, A. E., and Ho, Y. (1969). Applied Optimal Control (Blaisdell Publishing Company, Waltham, Mass.).

Chen, R. T. N., and Shen, D. W. C. (1969). Sensitivity analysis and the design of multivariable regulators using a quadratic performance criterion. Joint Automatic Control Conference, 229-237.

GRAUPE, D. (1972). Derivation of weighting matrics towards satisfying eigenvalue requirements. Int. J. Control, 16, 881-888.

Solneim, O. A. (1972). Design of optimal control systems with prescribed eigenvalues. Int. J. Control, 15, 142-160; (1979). Design of a class of optimal controllers via low-order Riccati equations. IFAC Symposium on Computer Aided Design of Control Systems. Zürich, Aug. 29-31, 1979.

TYler, J. S., and TUTEUR, F. B. (1966). The use of a quadratic performance index to design multivariable control systems. I.E.E.E. Trans. autom. Control, 11, 84-92.

VAN DE VEGTE, J., and MAKi, M. C. (1973). Optimization of systems with assigned poles. Int. J. Control, 18, 1105-1112.

WoOdhead, M. A., and Porter, B. (1973). Optimal control. Measurement and Control, 6, 301-303. 